\title{
A rare case of cerebral hemorrhage in a pre-term infant with coarctation of the aorta
}

\author{
Andreea Cerghit Paler ${ }^{1}$, Dorottya Miklosi', Liliana Gozar², \\ Rodica Toganel2 ${ }^{2}$, Amalia Fagarasan², Daniela Toma ${ }^{1}$ \\ ${ }^{1}$ Paediatric Cardiology, Emergency Institute for Cardiovascular Diseases and Transplantation, \\ Tg. Mures, Romania \\ ${ }^{2}$ Paediatric Cardiology, "George Emil Palade“ University of Medicine, Pharmacy, Science and Technology, \\ Tg. Mures, Romania
}

\begin{abstract}
Introduction. Coarctation of the aorta (CoA) occurs in 6-8\% of patients with congenital heart disease, with an incidence of 4/10,000 live births. It can be present with various clinical pictures, in an age range between newborn and adolescence. Infants with birth weights $\leq 2 \mathrm{~kg}$ with CoA have increased risk of severe intraventricular hemorrhage (IVH) compared to their birth weight-matched cohorts.

Case presentation. We present a case of a premature, male newborn, with low birth weight, who at the age of 3 weeks developed signs and symptoms of cardiogenic shock. Clinical examination, echocardiography and angio-CT confirmed the diagnosis of a severe CoA, without any flow through the ductus arteriosus. Treatment with prostaglandin $\mathrm{E} 1$ was initiated, to promote ductal patency. The systolic blood pressures were above 99 percentile for his gestational age in the upper extremities, with a noninvasive pressure gradient around $86 \mathrm{mmHg}$ between the arm and leg. Transfontanellar ultrasonography evaluation revealed a grade II/III bilateral IVH, in resorption, with cystic transformation. At the age of 6 weeks definitive surgical repair was performed. Postoperatively, at the 3 month follow-up, the clinical suspicion of recoarctation of the aorta arised, confirmed by echocardiography and angio-CT, with arterial hypertension and gradient around $37 \mathrm{mmHg}$ between the arm and leg. Prosthetic patch aortoplasty was performed. The postoperative evolution was marked by the persistence of arterial hypertension, the infant requiring continued treatment with angiotensin-converting enzyme inhibitor.

Conclusions. Premature neonates with low birth weight have always constituted a medical management challenge, with short and long term implications. A strong point in solving such cases is a multidisciplinary medical for assessment, management and outcomes.
\end{abstract}

Keywords: coarctation of the aorta, intraventricular hemorrage, prematurity, low birth weight

\section{INTRODUCTION}

Congenital heart diseases (CHD) accounts for nearly $28 \%$ of all major congenital anomalies [1].The birth incidence of CHD is estimated at 8/1,000 live births worldwide [2]. Coarctation af the aorta $(\mathrm{CoA})$ occurs in $6 \%$ to $8 \%$ of patients with CHD with an incidence of four per 10,000 live births [3]. It is more common in males and is the fourth most common lesion requiring cardiac catheterization or surgery during first year of life [4]. Coarctation of the aorta most frequently is a discrete stenosis in the upper thoracic aorta, at or near the insertion of ductus arteriosus. The aetiology of CoA has not yet been clearly identified.
There are two possible theories: one that includes abnormalities of blood flow in the aorta during fetal life, and another theory that includes excessive distribution of aberrant ductal tissue around the aortic isthmus [4]. Coarctation can occur in isolation, in association with bicuspid aortic valve or with major cardiac malformations [5]. It can present with different clinical pictures, depending on the severity of the narrowing of the aorta, from the neonatal period to adolescence [6]. The majority of coarctations are newly diagnosed in childhood, less than $25 \%$ are recognized beyond 10 years of age [5]. In the presence of clinical suspicion of aortic coarctation, echocardiography is the only available bedside diagnostic tool. It is not only used 
for the initial assessment, but also in the follow up of patients with coarctation after the surgical repair.

Regarding management of aortic coarctation in children, there are two methods of therapy. The first possibility consists of surgical correction, which is the standard method of therapy for CoA since 1940. Second one is transcatheter balloon angioplasty and it uses intravascular stents for management of coarctation, mainly in adolescents and adults [5].

The outcome after early repair of congenital heart diseases in low birth weight infants have improved. With regard CoA, two strategies have been adopted worldwide in the management of low birth weight (LBW) infants and neonates. One of that is delaying the timing of surgery until the baby grows up and gains weight; the other is in favor of early intervention. Many centers have reported low and acceptable surgical mortality rates, even in small infants [7]. Without treatment, the outcome for CoA patients is poor. Death may occur due to congestive heart failure, aortic dissection or rupture, endocarditis and intracranial bleeding [4].

Intraventricular hemorrhage (IVH) in children often causes death or lifelong disability. There is an association between the presence of CoA and intracranial hemorrhage, due either to chronic hypertension, co-existent cerebral aneurysms or a combination of the two. Premature and low birth weight infants have immature and more fragile cerebral vasculature, and have an increased risk for spontaneous intraventricular hemorrhage [6]. Infants with birth weights $\leq 2 \mathrm{~kg}$ with aortic coarctation have increased risk of severe IVH compared to their birth weight-matched cohorts [7].

\section{CLINICAL CASE}

\section{History}

We present a case of a male, premature newborn, born at a local center, at 34-35 gestational weeks, with low birth weight of 1,680 $\mathrm{g}$ and an APGAR score of 9 at 1 minute. We have no data regarding the pregnancy. From his personal history, we would like to mention that at the age of 3 weeks, the newborn became hemodynamically unstable, with marked respiratory syndrome, muco-tegumentary pallor, weakly perceptible pulse in the femoral arteries, with laboratory data being free from an inflammatory biological syndrome, requiring mechanical ventilation. A clinical diagnosis of aortic coarctation was made. Echocardiographic examination described signs of a severe aortic coarctation, with left ventricular hypertrophy and bicuspid aortic valve. Angio-CT showed aortic coarctation dis- tal from the emergence of the left subclavian artery, with a diameter of the isthmic zone of $4 \mathrm{~mm}$ (score $\mathrm{z}$ : -2.24) and post-stenotic dilatation, without any flow through the ductus arteriosus (PDA) (figure 1).

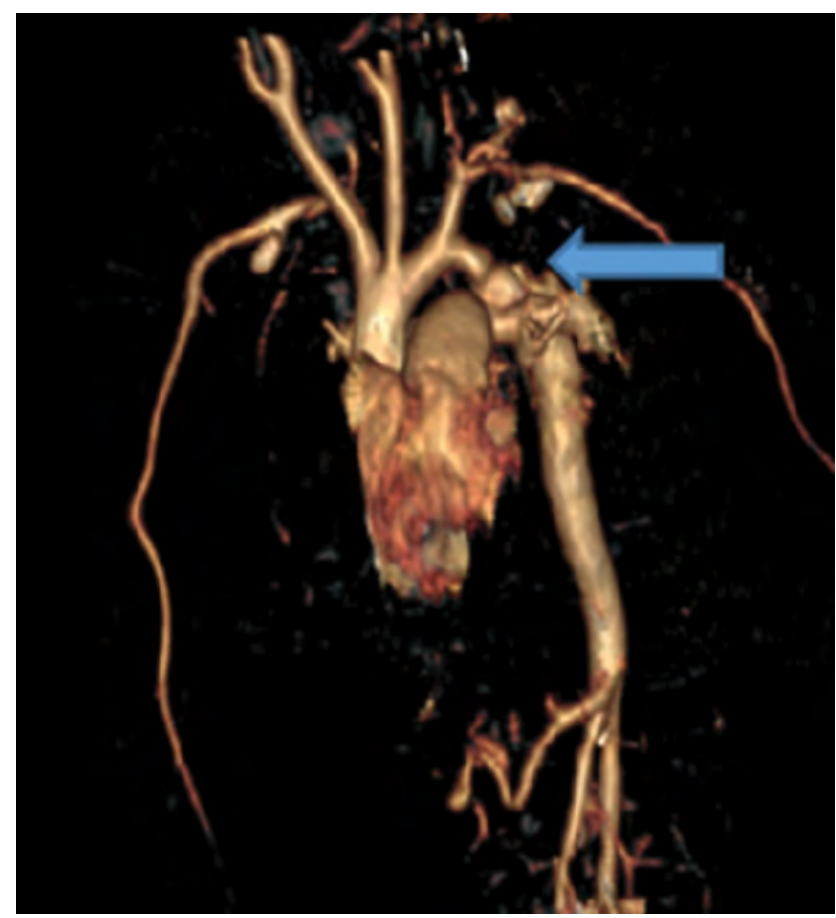

FIGURE 1. CT Angiography: Aortic coarctation distal from left subclavian artery emergence and post-stenotic dilatation

Prostaglandin E1 therapy was initiated to promote ductal patency and improve perfusion of the descending aorta, in association with diuretics and mechanic respiratory support - SIMV type was continued.

\section{Physical findings}

At the age of 6 weeks, the infant was transferred by helicopter ambulance to our clinic, in the intensive care unit (ICU). The state of the patient was critical. The child was pale, hypothermic, with diaphoresis, respiratory distress (moderate respiratory syndrome, oxygen saturation $96 \%$ ), and tachycardia, a grade $3 / 6$ systolic ejection murmur, best heard at the base and the left interscapular area posteriorly. He presented hepatomegaly and lower extremity pulses were absent. Conjunctival hemorrhage in the right eye was also observed, anterior fontanelle was in tension, dimensions around $6 / 7 \mathrm{~cm}$, with dehiscent cranial sutures, irritable status but with age appropriate response to stimuli, with mechanic ventilation and orotracheal intubation. The systolic blood pressures were elevated in the upper extremities (above 99 percentile for his gestational age [10]), with a noninvasive pressure gradient around $86 \mathrm{mmHg}$ between the arm (147/74 $\mathrm{mmHg})$ and leg $(61 / 42 \mathrm{mmHg})$. 


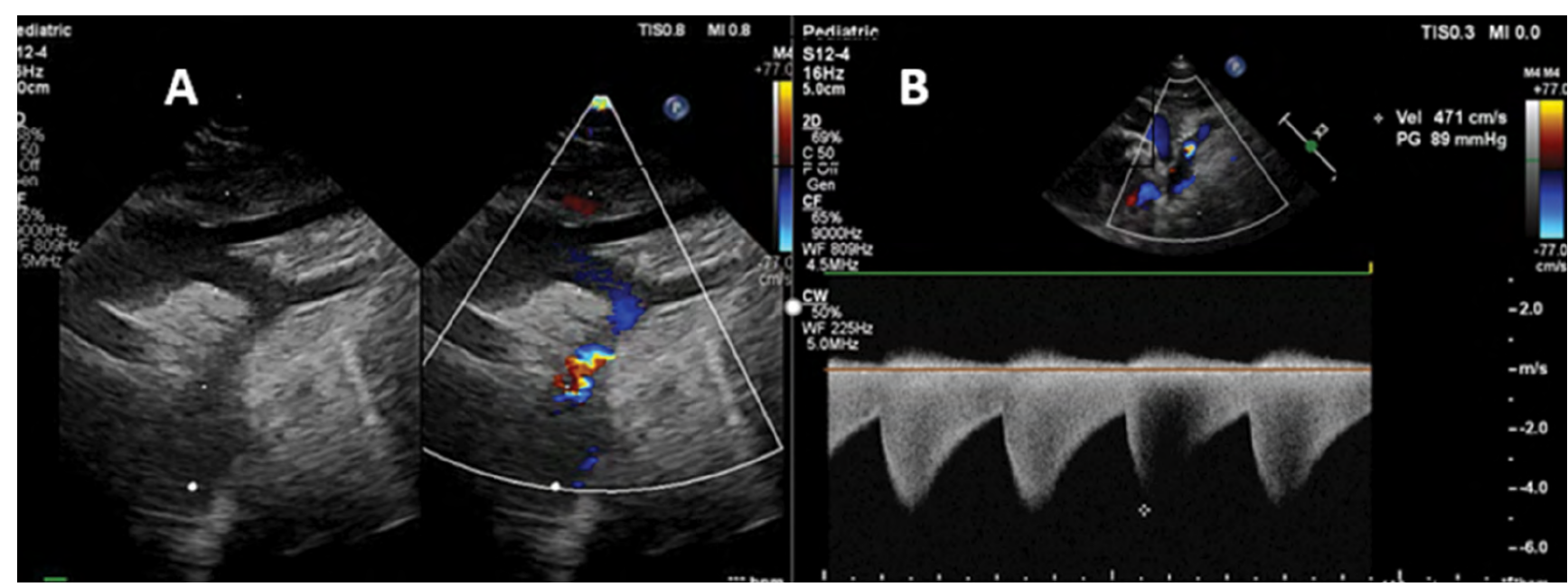

FIGURE 2. Ecocardiography suprasternal view: A - color compare image - Descending aorta coarctation, beyond the origin of the left subclavian artery, mild transverse arch hypoplasia. B - continuous wave Doppler with maxim velocity and gradient across coarctation site

\section{Paraclinical findings}

Laboratory test showed metabolic acidosis, hypopotassemia, anemia and mild hypoglycemia, without biological inflammatory syndrome, normal renal and hepatic function, no coagulopathy. The electrocardiogram reflected a sinus rhythm, heart rate 150 beats per minute, left ventricular hypertrophy and left atrial enlargement. The chest x-ray showed cardiomegaly (CTI: 0.61 ) and an abnormal contour of the aortic arch.

Two-dimensional and Doppler echocardiographic imaging study was performed. Echocardiography demonstrated the typical findings of a severe aortic coarctation: from the subcostal view, the abdominal aorta examined with pulsed Doppler showed a low flow rate, with the persistence of the gradient during diastole. The standard suprasternal view revealed that the narrowing of the descending aortic segment appears just below the origin of the left subclavian artery, mild hypoplasia of the transverse arch (z score -2.5 ) and a minimal patent ductus arteriosus. Continuous-wave Doppler in both systole and diastole was used to measure the maximum velocity across the coarctation site $(4.71 \mathrm{~m} / \mathrm{s}$ and $89 \mathrm{mmHg}$ gradient at this level). Also, a bicuspid, non-stenotic aortic valve and a small atrial septal defect was described. From the apical four chamber and parasternal long axis view, the assessment of ventricular size and function showed an important biventricular hypertrophy, a moderate systolic dysfunction (figure 2).

Considering the risk factors - the prematurity of the patient, the aspect of fontanelle and the elevated blood pressure -, a transfontanellar ultrasonography was performed. It revealed a grade II/III bilateral intraventricular hemorrhage, in resorption, with cystic transformation (figure 3).

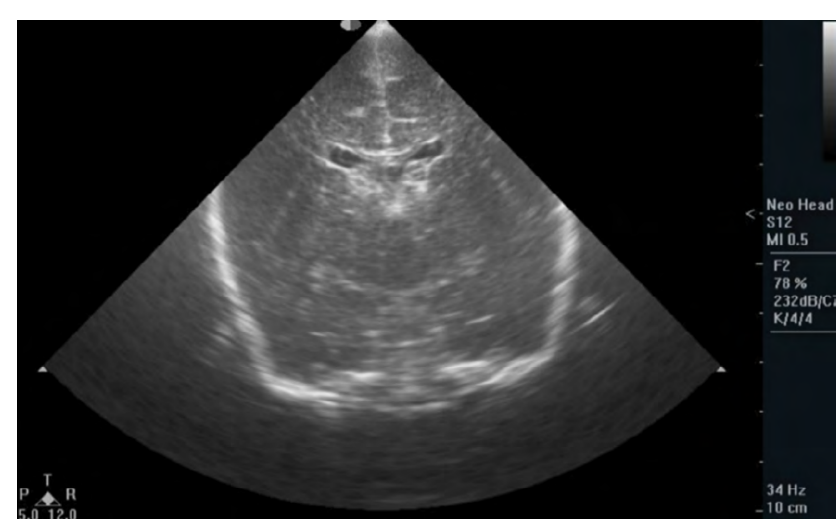

FIGURE 3. Transfontanellar ultrasonography with bilateral intraventricular hemorrhage, with cystic transformation

The neurological consultant established the diagnosis of perinatal hypoxic lesion, hypotonic syndrome and intraventricular hemorrhage- in resorption. Neuro-roborant and depletive treatment was recommended. The patient associates sacral dermal sinus for which the neurosurgical consultant recommended lumbosacral MRI, which for technical reason was unavailable at that time.

\section{Diagnosis}

At that moment, we established the following diagnosis: severe coarctation of the isthmic aorta with mild transverse arch hypoplasia, restrictive PDA, bicuspid non-stenotic aortic valve, small ASD, secondary high blood pressure, intraventricular hemorrhage in resorption, perinatal hypoxic lesion, hypotonic syndrome and prematurity.

\section{Treatment}

After a brief period of medical management to stabilize the child on the ICU (correction of the meta- 
bolic disturbance - acidosis, hypothermia, hypoglycemia) definitive surgical repair was performed (consisting in resection of stenotic segment and endto-end anastomosis, heterologous pericardial patch aortoplasty, ligation of the ductus arteriosus with left latero-thoracic surgical approach).

\section{Postoperative evolution. Complications}

In the postoperative period, the patient presented high blood pressure values for gestational age, requiring drug tratment with angiotensin-converting enzyme (ACE) inhibitor and diuretics. The arterial hypertension was not controled even with maximal dosage, so the ACE inhibitor was changed to betablocker medication with the maintainance of the diuretic. Thus a favorable therapeutic response was achieved. Serial postoperative echocardiographies showed a residual systolic gradient at aortic isthmus of $20 \mathrm{mmHg}$. Transfontanellar echography was without signs of intracranial hypertension or acute intraventricular hemorrage.

Postoperative evolution was slowly favorable. On the 18th postoperative day the patient was discharged in a good general condition, hemodynamically stable, oxygen saturation of $99 \%$, heart rate $130 \mathrm{~b} / \mathrm{min}$, in sinus rhythm, without hepatomegaly, ascending weight curve and good digestive tolerance, controlled blood pressure $(74 / 41 \mathrm{mmHg}$ ) with beta-blocker (Propranolol $1.6 \mathrm{mg} / \mathrm{kg} / \mathrm{d}$ ) and low doses of diuretics (spironolactone and hydrochlorothiazide). The neurologic status was also improved.

\section{3 months follow up}

At the 3 months follow-up, the infant was in an altered clinical status. Upon clinical evaluation the patient was pale, with a wide, normotensive anterior fontanelle, mild/moderate respiratory syndrome, oxy- gen saturation 97\%, tachycardia, hepatomegaly, diminished femoral pulses, but with good digestive tolerance. The systolic blood pressures were elevated in the upper extremities (above 99 percentile for his gestational age), with a systolic pressure gradient around $37 \mathrm{mmHg}$ between the arm $(142 / 73 \mathrm{mmHg})$ and leg $(105 / 87 \mathrm{mmHg})$. Thus the suspicion of a recoarctation of the aorta arise, confirmed later on by echocardiographic evaluation (the restenosis of aortic isthmus and continuous-wave Doppler with high flow velocity $4.34 \mathrm{~m} / \mathrm{s}, 75 \mathrm{mmHg}$ gradient at this level) and angioCT (aortic isthmus recoarctation with a diameter of $1.2 \mathrm{~mm}$ ) (Figure 4).

Under these conditions: high blood pressure and recoarctation, the infant was neurologically reassessed, with no pathological changes compared to the previous evaluation, being without signs of intracranial hypertension or acute bleeding.

Pending the surgical reintervention, due to the elevated blood pressure levels (over 99th percentile), the dose of beta-blocker medication with Propranolol ( $1.6 \mathrm{mg} / \mathrm{kgc} /$ day) was readjusted and ACE inhibitor (Captopril) was associated.

After a brief period, prosthetic patch aortoplasty was performed. Postoperative echocardiographic evaluations showed a residual systolic gradient of 19 $\mathrm{mmHg}$. The postoperative evolution was marked by the persistence of the high blood pressure values, the infant requiring continued treatment with $\mathrm{ACE}$ inhibitor, recommended treatment including at discharge.

\section{DISCUSSIONS}

In aortic coarctation, based on patient's age, the clinical presentation and exam findings are variable, typically an earlier presentation corresponds to a more severe disease. Newborns and neonates are usually

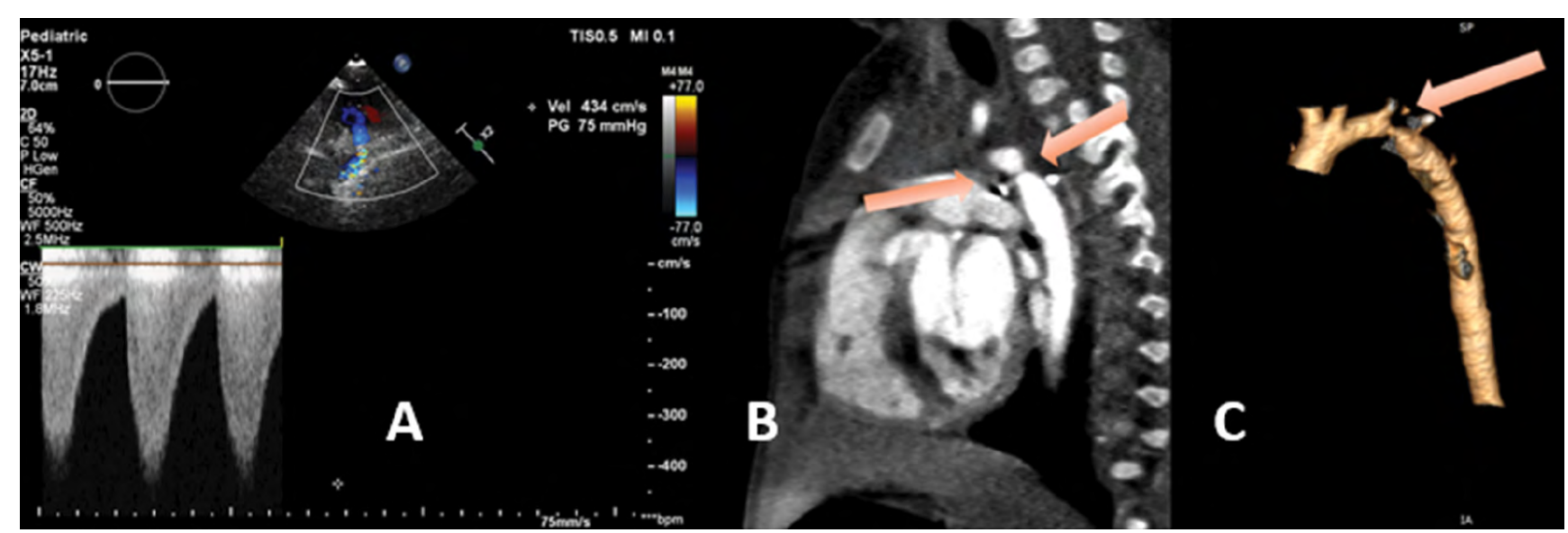

FIGURE 4. A - Ecocardiography suprasternal view, continuous wave Doppler with maxim velocity and gradient across coarctation site. $B$ - Thoracic angiography $C T$, sagital view of the coarctation site. $C-$ Aorta view from $3 D$ angio $C T$ 
asymptomatic right after birth as patent ductus arteriosus helps perfuse lower body irrespective of severity of CoA [4]. Our patient, with severe CoA, developed signs and symptoms of cardiogenic shock as the ductus arteriosus was closed.

Mehwald et al. described a similar case of a previously well 31 day old boy, with agitation, tachypnea, opistotonus, tensed fontanel, not palpable femoral pulses and blood pressure gradient between the upper and lower limb of $100 \mathrm{mmHg}$. Cerebral hemorrhage was found on ultrasound examination and echocardiography showed severe coarctation of the aorta. Surgical repair of the aortic coarctation was performed and a ventriculoperitoneal shunt was needed because of an intracerebral hematoma with high cerebral pressure [11].

The most likely cause of cerebral hemorrhage in neonates is an arteriovenous malformation, followed by aortic coarctation. There are several possible causes of increased risk of IVH in the presence of aortic coarctation [9]. The first risk factor identifed by several studies is prolonged exposure to upper extremity hypertension. Premature and low birth weight infants have immature and more fragile cerebral vasculature, and are at increased risk for spontaneous intraventricular hemorrhage. The additional stress made by systemic artherial hypertension on these vessels may make them more likely to hemorrhage. A second risk factor is the need to maintain the patency of the ductus arteriosus, thus leading to cerebral ischemia caused by left-to-right shunting through the PDA. A third risk factor may be treatment of the patent ductus arteriosus with PGE1 which can induce IVH through platelet dysfunction [12]. Our patient presented not only prematurity and LBW, but also aortic coarctation with high systemic blood presure. Due to the fact that a cranial CT examination was not performed, a cerebral aneurysm could not be excluded. Delaying the diagnosis and the surgical correction of the CoA only prolongs the exposure to these risk factors for IVH.

Young et al. described two similary cases of preterm male newborns in whom the presence of neurological signs and markerd systemic hypertension led to the diagnosis of CoA. They drew attention that the diagnosis of aortic coarctation may be overlooked in infants with cerebral hemorrhage if there is not a high degree of suspicion [13].

It is generally considered that low birth weight is not a contraindication for early repair of congenital cardiac anomalies. High mortality rates have been reported in small infants with uncorrected CoA. Sudarshan $\mathrm{CD}$ et al. retrospectively reviewed twenty-four consecutive babies weighing $2 \mathrm{~kg}$ or less who were operated for CoA on over a period of 15 years [7]. The methods of choice was surgical resection of the narrowed zone. Recoarctation developed in 7 babies. Risk factor analyses revealed that the presence of preoperative congestive cardiac failure, and coexisting noncardiac lesions as well as the duration of descending aortic cross-clamp and postoperative ventilation had a significant influence on mortality after repair. Moreover, McElhinney et al. concluded that low weight was not a risk factor for recurrent stenosis after repair of CoA in small infants [14]. However, Bacha et al. found that low birth weight (less than $1.5 \mathrm{~kg}$ ) infants had a significantly higher chance of having residual CoA or developing recoarctation of the aorta [15].

In our preterm and LBW patient, first surgical intevention was earlier than two months of life and recoarctation developed earlier than three months from the first surgery.

Baykan et al. reported three hypertensive children with stroke in association with undetected and untreated CoA. They concluded that occult aortic coarctation should be suspected in any young hypertensive patients, especially in those with cerebrovascular syndromes. If a pediatric patient has hypertension, further examination must be done for etiology, and causes of secondary hypertension must be evaluated [6].

Long-term survival after CoA repair remains lower than in the general population, mainly due to the long-term effects of persistent systemic arterial hypertension [4]. In addition, intraventricular hemorrhage is associated with poor functional outcomes [16].

\section{CONCLUSIONS}

Premature neonates with low birth weight have always constituted a medical management challenge, with short and long term implications. In the case reported, we presented a premature neonate, born with low birth weight and diagnosed with critical coarctation of aorta. We exposed the challenges faced in the process of stabilizing the neonate. In such cases of LBW premature, associated with CoA and it's surgical repair, there is a high risk of recoarctation, secondary hypertension and cerebral hemorrhage. Our conclusions might help practitioners predict postoperative complications and optimize therapeutic strategies and also the postoperative evolution in intensive care unit. A strong point in solving such cases is a multidisciplinary medical for assessment, management and outcomes. 


\section{REFERENCES}

1. Dolk H, Loane M, Garne E. European Surveillance of Congenital Anomalies (EUROCAT) Working Group. Congenital heart defects in Europe: prevalence and perinatal mortality, 2000 to 2005 . Circulation. 2011;123(8):841-849.

2. Bernier PL, Stefanescu A, Samoukovic G, Tchervenkov Cl. The challenge of congenital heart disease worldwide: Epidemiologic and demographic facts. Semin Thorac Cardiovasc Surg Pediatr Card Surg Annu. 2010;13(1):26-34.

3. Mason CA, Kirby RS, Sever LE, Langlois PH. Prevalence is the preferred measure of frequency of birth defects. Birth Defects Res A Clin Mol Teratol. 2005;73(10):690-692.

4. Beekman R. Coarctation of the Aorta. In: Allen H, Shaddy E, Penny F, Feltes T, Cetta F. Moss and Adams' Heart Disease in Infants, Children, and Adolescents: including the fetus and young adult. 9th ed. Lippincott Williams \& Wilkins; 2016:1107-1125.

5. Doshi A, Rao S. Coarctation of aorta-management options and decision making. Pediat Therapeut. 2012;S5-006.

6. Baykan A, Argun M, Ozyurt A, Pamukçu O, Uzüm K, Narın N. Hypertension associated with coarctation of the aorta revisited: Case-based update from experience of three children. Case Rep Pediatr. 2013;2013:716438.

7. Sudarshan CD, Cochrane AD, Jun ZH, Soto R, Brizard CP. Repair of coarctation of the aorta in infants weighing less than 2 kilograms. Ann Thorac Surg. 2006;82(1):158-163.

8. Stoll BJ, Hansen NI, Bell EF et al. Neonatal outcomes of extremely preterm infants from the NICHD Neonatal Research Network. Pediatrics. 2010;126(3):443-456.
9. Seckeler MD, White SC, Fox KA. Increased risk of intraventricular hemorrhage in low birth weight infants with aortic coarctation. J Matern Fetal Neonatal Med. 2020;33(8):1273-1275.

10. Nickavar A, Assadi F. Managing hypertension in the newborn infants. Int J Prev Med. 2014;5(Suppl 1):S39-S43.

11. Mehwald PS, Dittrich S, Grohmann J, Bley T, Kececioglu D. Coarctation of the aorta presenting as cerebral hemorrhage. J Pediatr. 2005;146(2):293.

12. Brunner B, Hoeck M, Schermer E, Streif W, Kiechl-Kohlendorfer U. Patent ductus arteriosus, low platelets, cyclooxygenase inhibitors, and intraventricular hemorrhage in very low birth weight preterm infants. J Pediatr. 2013;163(1):23-28.

13. Young RS, Liberthson RR, Zalneraitis EL. Cerebral hemorrhage in neonates with coarctation of the aorta. Stroke. 1982;13(4):491-494.

14. McElhinney DB, Yang SG, Hogarth AN et al. Recurrent arch obstruction after repair of isolated coarctation of the aorta in neonates and young infants: Is low weight a risk factor? J Thorac Cardiovasc Surg. 2001;122:883-90.

15. Bacha EA, Almodovar M, Wessel DL et al. Surgery for coarctation of the aorta in infants weighing less than $2 \mathrm{~kg}$. Ann Thorac Surg. 2001;71(4):1260-1264.

16. Li Q, Huang YJ, Zhang G et al. Intraventricular Hemorrhage and Early Hematoma Expansion in Patients with Intracerebral Hemorrhage. Sci Rep. 2015;5:11357. 